\title{
Multi-Pion Correlation Effects on Two-Pion Interferometry *
}

\author{
W.Q. Chao ${ }^{a, b, c}$, C.S. Gao ${ }^{a, c, d}$ and Q.H. Zhang ${ }^{a}$ \\ a China Center of Advanced Science and Technology, (World Laboratory) P.O. Box 8730, Beijing \\ 100080, China \\ b Institute of High Energy Physics, Academia Sinica, P.O. Box 918(4), Beijing 100039, China \\ c Institute of Theoretical Physics, Academia Sinica, P.O. Box 2735, Beijing 100080, China \\ d Physics Department, Peking University, Beijing 100871, China
}

\begin{abstract}
A general derivation of the multi-pion correlation function for completely chaotic source is given. Its effects on the pion multiplicity distribution, twopion interferometry are studied. A generalized multi-pion correlation function for a partially coherent source is also discussed.
\end{abstract}

\section{INTRODUCTION}

Hanbury-Brown and Twiss [1] were the first who applied the Bose-Einstein correlations to measure the size of distant stars. The method was first applied to particle physics by Goldhaber et al. [0] in 1959. Since then the size of the interaction region has been measured by numerous experiments in high energy collisions using many different types of particles [3 6]. The basic idea of HBT (GGLP) effect is the following: The intensity correlation function of the two identical particles in momentum space is related to the Fourier transformation of the space-time distribution of the source. Therefore using the two particle correlation function in momentum space, one can extract the radius and lifetime

\footnotetext{
* Partly supported by the National Science Foundation of China
} 
of the source. The source size measurement in high energy heavy-ion collisions has its special meaning: Knowing the size of the source and the stored energy in it one can obtain the corresponding energy density reached in the event, which is one of the key quantities to investigate the possible transition to quark-gluon plasma (QGP) phase in high energy heavy-ion collisions.

In principle, the extension of such methods to more than two pions is straightforward [7-14]. Experimentally, ultrarelativistic hadronic and nuclear collisions provide the environment for creating dozens and in some cases hundreds of pions 15 18. Therefore, it is necessary to consider the multi-pion correlations in these processes. The Bosonic nature of pion should affect the single and two pion spectrum and distort the two-pion correlation function. So it is very interesting to analyze the effects of multi-pion correlation on these variables 9]. Actually the bosonic nature also causes the abundance of pion at small momentum. Therefore, people are also interested in analyzing the effects of multi-pion Bose-Einstein correlation on the multiplicity distribution [19.20].

In 1987, based on Monte-Carlo methods, Zajc analysed the effects of multi-pion correlation on the phase-space and two-pion interferometry [9]. Based on T matrix methods, Pratt analysed the effects of multi-pion correlation on the pion multiplicity distribution [20]. In our paper, we give a more general derivation of the effects of multi-pion correlation on two-pion interferometry. The arrangement of this paper is as follows: The derivation of the multi-pion correlation function for totally chaotic sources is given in section 2. In section 3 , the effects of multi-pion correlation on the multiplicity distribution are discussed. In section 4, the effects of multi-pion correlation on the two-pion correlation function are analysed both for $\mathrm{N}$ pion events and for events with all possible multiplicities. In section $5, \mathrm{n}$ pion correlation function for a partially coherent source is derived. In section 6, as an example, our results for a special source distribution are given, together with some discussions. 


\section{N-PION CORRELATION FUNCTION FOR A CHAOTIC SOURCE}

The general definition of the N-pion correlation function $C_{n}\left(\vec{p}_{1}, \cdots \vec{p}_{n}\right)$ is

$$
C_{n}\left(\vec{p}_{1}, \cdots \vec{p}_{n}\right)=\frac{P_{n}\left(\vec{p}_{1}, \cdots, \vec{p}_{n}\right)}{\prod_{i=1, n} P_{1}\left(\vec{p}_{i}\right)}
$$

where $P_{n}\left(\vec{p}_{1} \ldots \vec{p}_{n}\right)$ is the probability of observing $n$ pions with momenta $\left\{\vec{p}_{i}\right\}$ all in the same event. Here we derive a formula for the correlation function which is valid for a totally chaotic source.

A state created by a classical pion source is described by [6,21]

$$
\left|\phi>=\exp \left(i \int d \vec{p} \int d^{4} x j(x) \exp (i p x) c^{+}(\vec{p})\right)\right| 0>
$$

where $c^{+}(\vec{p})$ is the pion creation operator. $j(x)$ is the current of the pion, which can be expressed as

$$
j(x)=\int d^{4} x^{\prime} d \vec{p} A\left(x^{\prime}\right) j\left(x^{\prime}, \vec{p}\right) \nu\left(x^{\prime}\right) \exp \left(i p\left(x-x^{\prime}\right)\right)
$$

here $j\left(x^{\prime}, \vec{p}\right)$ is the probability amplitude of finding a pion with momentum $\vec{p}$, emitted by the source at $x^{\prime} . A\left(x^{\prime}\right)$ is the probability amplitude of the source distribution. $\nu\left(x^{\prime}\right)$ is a random phase factor. All sources are uncorrelated in coordinate space when assuming:

$$
<\nu^{*}\left(x^{\prime}\right) \nu(x)>=\delta^{4}\left(x^{\prime}-x\right)
$$

The coherent state can expand in Fock-Space as

$$
\left|\phi>=\sum_{n=0, \infty} \frac{\left(i \int j(x) e^{i p x} c^{+}(p) d \vec{p} d x\right)^{n}}{n !}\right| 0>=\sum_{n=0, \infty} \mid n>
$$

with

$$
\left|n>=\frac{\left(i \int j(x) e^{i p x} c^{+}(p) d \overrightarrow{p d} x\right)^{n}}{n !}\right| 0>
$$

Then we define the n-pion correlation function for n-pion state

$$
C_{n}\left(\vec{p}_{1}, \ldots . \vec{p}_{n}\right)=\frac{<n\left|c^{+}\left(p_{1}\right) \cdots c^{+}\left(p_{n}\right) c\left(p_{n}\right) \cdots c\left(p_{1}\right)\right| n>}{\prod_{i=1, n}<1\left|c^{+}\left(p_{i}\right) c\left(p_{i}\right)\right| 1>}
$$


From above definition, the two-pion correlation function for two-pion state can be written as

$$
\begin{aligned}
C\left(\vec{p}_{1}, \vec{p}_{2}\right) & =\frac{<2\left|c^{+}\left(\vec{p}_{1}\right) c^{+}\left(\vec{p}_{2}\right) c\left(\vec{p}_{2}\right) c\left(\vec{p}_{1}\right)\right| 2>}{<1\left|c^{+}\left(\vec{p}_{1}\right) c\left(\vec{p}_{1}\right)\right| 1><1\left|c^{+}\left(\vec{p}_{2}\right) c\left(\vec{p}_{2}\right)\right| 1>} \\
& =1+\frac{<1 \mid c^{+}\left(\vec{p}_{1}\right) c\left(\vec{p}_{2}|1><1| c^{+}\left(\vec{p}_{2}\right) c\left(\vec{p}_{1}\right) \mid 1>\right.}{<1\left|c^{+}\left(\vec{p}_{1}\right) c\left(\vec{p}_{1}\right)\right| 1><1\left|c^{+}\left(\vec{p}_{2}\right) c\left(\vec{p}_{2}\right)\right| 1>}
\end{aligned}
$$

Noticing that

$$
c(\vec{p})\left|n>=i \int d^{4} x j(x) \exp (i p x)\right| n-1>,
$$

using eq.(3), we have

$$
\begin{aligned}
<1\left|c^{+}\left(\vec{p}_{1}\right) c\left(\vec{p}_{2}\right)\right| 1>= & \int d^{4} x_{1} d^{4} x_{2} j^{*}\left(x_{1}\right) j\left(x_{2}\right) \exp \left(-i\left(p_{1} x_{1}-p_{2} x_{2}\right)\right) \\
= & \int d^{4} x_{1}^{\prime} d^{4} x_{2}^{\prime} j^{*}\left(x_{1}^{\prime}, \vec{p}_{1}\right) j\left(x_{2}^{\prime}, \vec{p}_{2}\right) \nu^{*}\left(x_{1}^{\prime}\right) \nu\left(x_{2}^{\prime}\right) \\
& A^{*}\left(x_{1}^{\prime}\right) A\left(x_{2}^{\prime}\right) \exp \left(-i p_{1} x_{1}^{\prime}+i p_{2} x_{2}^{\prime}\right) .
\end{aligned}
$$

Taking the phase average, we get

$$
<1\left|c^{+}\left(\vec{p}_{1}\right) c\left(\vec{p}_{2}\right)\right| 1>=\int d^{4} x_{1}^{\prime} j^{*}\left(x_{1}^{\prime}, p_{1}\right) j\left(x_{1}^{\prime}, p_{2}\right)\left|A\left(x_{1}^{\prime}\right)\right|^{2} \exp \left(-i\left(p_{1}-p_{2}\right) x_{1}^{\prime}\right)
$$

We define the semiclassical function

$$
g\left(x^{\prime}, x, k\right)=\int d^{4} q j^{*}\left(x^{\prime}, k+q / 2\right) j\left(x^{\prime}, k-q / 2\right) \exp \left(i q\left(x-x^{\prime}\right)\right) .
$$

Here $k, q$ are four dimensional momenta. $g\left(x^{\prime}, x, k\right)$ can be explained as the probability of finding a pion with momentum $k$ at point $x$ due to the source at point $x^{\prime}$. Then we have

$$
j^{*}\left(x^{\prime}, k+q / 2\right) j\left(x^{\prime}, k-q / 2\right)=\int d^{4} x g\left(x^{\prime}, x, k\right) \exp \left(-i q\left(x-x^{\prime}\right)\right),
$$

and eq.(10) becomes

$$
<1\left|c^{+}\left(\vec{p}_{1}\right) c\left(\vec{p}_{2}\right)\right| 1>=\int d^{4} x g(x, k) \exp (-i q x)
$$


Here $k=\frac{1}{2}\left(p_{1}+p_{2}\right), q=p_{1}-p_{2}$ and $g(x, k)$ is the probability of finding a pion with momentum $k$ at point $x$. It is defined as

$$
g(x, k)=\int d^{4} x^{\prime} \rho\left(x^{\prime}\right) g\left(x^{\prime}, x, k\right)
$$

where

$$
\rho(x)=A^{*}(x) A(x)
$$

is the source distribution probability. Inserting eq. (11) into eq. (5) gives the correlation function

$$
C\left(\vec{p}_{1}, \vec{p}_{2}\right)=1+\frac{\int d^{4} x d^{4} x^{\prime} g(x, k) g\left(x^{\prime}, k\right) \exp \left(i q\left(x-x^{\prime}\right)\right)}{\int d^{4} x d^{4} x^{\prime} g\left(x, p_{1}\right) g\left(x^{\prime}, p_{2}\right)},
$$

where $q=p_{1}-p_{2}, k=\left(p_{1}+p_{2}\right) / 2$.

Similarly, the $n$-pion correlation function can be expressed as

$$
C_{n}\left(p_{1}, p_{2}, \cdots p_{n}\right)=\sum_{\sigma} \chi_{1, \sigma(1)} \chi_{2, \sigma(2)} \cdots \chi_{n, \sigma(n)}
$$

with the expression of $\chi_{i, j}$ as

$$
\chi_{i, j}=\chi\left(p_{i}, p_{j}\right)=\frac{\int d^{4} x g\left(x, \frac{\left(p_{i}+p_{j}\right)}{2}\right) e^{i\left(p_{i}-p_{j}\right) x}}{\sqrt{\int d^{4} x d^{4} y\left(g\left(x, p_{i}\right) g\left(y, p_{j}\right)\right.}} .
$$

In the following, we often use the expression of the n-pion momentum probability distribution $S_{n}$ which can be expressed as

$$
S_{n}=\sum_{\sigma} \rho_{1, \sigma(1)} \rho_{2, \sigma(2)} \ldots \rho_{n, \sigma(n)}
$$

with

$$
\rho_{i, j}=\rho\left(p_{i}, p_{j}\right)=\frac{1}{n_{0}} \int d^{4} x g\left(x, \frac{\left(p_{i}+p_{j}\right)}{2}\right) e^{i\left(p_{i}-p_{j}\right) x}
$$

Here $n_{0}$ is the mean multiplicity without B-E correlation, defined as

$$
n_{0}=\int g(x, k) d^{4} x d^{4} k
$$


$\sigma(i)$ denotes the $i$ th element of a permutation of the sequence $1,2,3, \cdots, n$, and the sum over $\sigma$ denotes the sum over all $n$ ! permutations of this sequence.

Once the source distribution $\mathrm{g}(\mathrm{x}, \mathrm{k})$ is known, the multi-pion correlation function can be calculated from eq.(18). Therefore, $C_{n}$ can also expressed by $S_{n}$ as

$$
C_{n}\left(p_{1}, p_{2}, \cdots, p_{n}\right)=\frac{S_{n}\left(p_{1}, p_{2}, \cdots, p_{n}\right)}{\prod_{i} \rho_{i, i}}
$$

\section{EFFECTS OF MULTI-PION CORRELATION ON PION MULTIPLICITY DISTRIBUTION}

From the above definition of $|n\rangle$, we have

$$
\begin{aligned}
\omega(n)=<n \mid n>= & \frac{1}{n !}<0 \mid \int j^{*}\left(x_{1}\right) e^{-i p_{1} x_{1}} d x_{1} \int j\left(x_{2}\right) e^{i p_{1} x_{2}} d x_{2} d p_{1} \\
& \cdots \int j^{*}\left(x_{2 n-1}\right) e^{-i p_{n} x_{2 n-1}} d x_{2 n-1} \int j\left(x_{2 n}\right) e^{i p_{n} x_{2 n}} d x_{2 n} d p_{n} \mid 0>,
\end{aligned}
$$

Following a similar derivation as in section two, we get

$$
\omega(n)=<n \mid n>=\frac{n_{0}^{n}}{n !} \int S_{n} \prod_{i=1, n} d \vec{p}_{i} .
$$

Then we have

$$
<\phi\left|\phi>=\sum_{k}<k\right| k>=\sum_{k} \frac{n_{0}^{k}}{k !} \int d \vec{p}_{1} \cdots d \vec{p}_{k} S_{k} .
$$

After considering the effect of multi-pion Bose-Einstein correlation, the multiplicity distribution of pion becomes

$$
P(n)=\frac{<n \mid n>}{<\phi \mid \phi>}=\frac{\omega(n)}{\sum_{k} \omega(k)} .
$$

For particles without BE Correlation, we have

$$
\rho_{i, j}=\delta_{i, j} \rho_{i, i}
$$

therefore, 


$$
\int S_{n} \prod_{i} d \vec{p}_{i}=1
$$

and $P(n)$ reduces to the usual Poisson distribution. From above equation, we can also get the mean multiplicity $<M>$

$$
<M>=\sum_{n} n \cdot P(n)
$$

\section{EFFECTS OF MULTI-PION CORRELATION ON TWO-PION CORRELATION FUNCTION}

\section{A. Two-pion correlation function for n-pion events}

For $n \pi$ events, the two-pion correlation function can be defined as

$$
C_{2}^{n}=\frac{P_{2}^{n}\left(\vec{p}_{1}, \vec{P}_{2}\right)}{P_{1}^{n}\left(\vec{p}_{1}\right) P_{1}^{n}\left(\vec{p}_{2}\right)}
$$

where $P_{2}^{n}\left(\vec{p}_{1}, \vec{p}_{2}\right)$ is the modified two-pion inclusive distribution in $n$ pion events. The definition of $P_{i}^{n}\left(\vec{p}_{1}, \cdots, \vec{p}_{i}\right)$ is

$$
P_{i}^{n}\left(\vec{p}_{1}, \cdots \vec{p}_{i}\right)=\frac{<n\left|c^{+}\left(\vec{p}_{i}\right) \cdots c^{+}\left(\vec{p}_{1}\right) c\left(\vec{p}_{1}\right) \cdots c\left(\vec{p}_{i}\right)\right| n>}{<n \mid n>n \cdots(n-i+1)}
$$

For an event with multiplicity of $n$, the two-pion inclusive and single-pion inclusive distribution can be expressed as

$$
P_{2}^{n}\left(\vec{p}_{1}, \vec{p}_{2}\right)=\frac{\int \prod_{i=3, n} d \vec{p}_{i} S_{n}\left(\vec{p}_{1} \ldots \vec{p}_{n}\right)}{\int \prod_{i=1, n} d \vec{p}_{i} S_{n}\left(\vec{p}_{1} \ldots \vec{p}_{n}\right)}
$$

and

$$
P_{1}^{n}\left(\vec{p}_{1}\right)=\frac{\int \prod_{i=2, n} d \vec{p}_{i} S_{n}\left(\vec{p}_{1} \ldots \vec{p}_{n}\right)}{\int \prod_{i=1, n} d \vec{p}_{i} S_{n}\left(\vec{p}_{1} \ldots \vec{p}_{n}\right)}
$$

As $n$ increases, the calculation of the intergration given above becomes more and more complex. For the two-pion inclusive distribution in the $n$ pion events, there are only twokinds of terms that we are interested in. One is 


$$
\cdots \rho\left(p_{1}, p_{k}\right) \rho\left(p_{k}, p_{l}\right) \cdots \rho\left(p_{n 1}, p_{n 2}\right) \rho\left(p_{n 2}, p_{2}\right) \rho\left(p_{2}, p_{m}\right) \rho\left(p_{m}, p_{n 3}\right) \cdots \rho\left(p_{n 4}, p_{i}\right) \rho\left(p_{i}, p_{1}\right) \cdots
$$

the other is

$$
\cdots \rho\left(p_{1}, p_{k}\right) \rho\left(p_{k}, p_{l}\right) \cdots \rho\left(p_{j}, p_{1}\right) \rho\left(p_{2}, p_{l}\right) \rho\left(p_{l}, p_{i 1}\right) \cdots \rho\left(p_{m}, p_{2}\right) \cdots
$$

Now we define the function

$$
G_{i}(p, q)=n_{0}^{i} \int \rho\left(p, p_{1}\right) d \vec{p}_{1} \rho\left(p_{1}, p_{2}\right) d \vec{p}_{2} \cdots \rho\left(p_{i-2}, p_{i-1}\right) d \vec{p}_{i-1} \rho\left(p_{i-1}, q\right) .
$$

The intergration in above term can be expressed as

$$
\begin{aligned}
& \int \rho\left(p, p_{1}\right) d \vec{p}_{1} \rho\left(p_{1}, p_{2}\right) d \vec{p}_{2} \cdots d \vec{p}_{k-1} \rho\left(p_{k-1}, q\right) \\
& \rho\left(q, p_{k+1}\right) d \vec{p}_{k+1} \rho\left(p_{k+1}, p_{k+2}\right) d \vec{p}_{k+2} \cdots d \vec{p}_{k+i-1} \rho\left(p_{k+i-1}, p\right) \\
& =G_{k}(p, q) \cdot G_{i}(q, p)
\end{aligned}
$$

and

$$
\begin{aligned}
& \int \rho\left(p, p_{1}\right) d \vec{p}_{1} \rho\left(p_{1}, p_{2}\right) d \vec{p}_{2} \cdots d \vec{p}_{i-1} \rho\left(p_{i-1}, p\right) \\
& \rho\left(q, p_{j+1}\right) d \vec{p}_{j+1} \rho\left(p_{j+1}, p_{j+2}\right) d \vec{p}_{j+2} \cdots d \vec{p}_{j+m-1} \rho\left(p_{j+m-1}, q\right) \\
& =G_{i}(p, p) G_{m}(q, q) .
\end{aligned}
$$

From the expression of $S_{n}$

$$
S_{n}=\sum_{\sigma} \rho_{1, \sigma(1)} \rho_{2, \sigma(2)} \ldots \rho_{n, \sigma(n)}
$$

the number of terms of the form

$$
\rho\left(p_{1}, p_{k}\right) \rho\left(p_{k}, p_{j}\right) \rho\left(p_{j}, p_{l}\right) \cdots \rho\left(p_{m}, p_{1}\right) \rho\left(p_{2}, p_{i 1}\right) \rho\left(p_{i 1}, p_{i m}\right) \rho\left(p_{i m}, p_{i n}\right) \cdots \rho\left(p_{i k}, p_{2}\right) C_{n-i}
$$


is

$$
\frac{(n-2) !}{(n-i) !}
$$

$i$ is the number of $\rho$ that has not been included in $C_{n-i}$. Similarly the number of terms of the form

$$
\rho\left(p_{1}-p_{k}\right) \rho\left(p_{k}-p_{l}\right) \cdots \rho\left(p_{j}-p_{2}\right) \rho\left(p_{2}-p_{m}\right) \cdots \rho\left(p_{i 1}-p_{1}\right) C_{n-i}
$$

is also

$$
\frac{(n-2) !}{(n-i) !}
$$

Then the two-pion inclusive distribution can be expressed as

$$
P_{2}^{n}(\vec{p}, \vec{q})=\frac{1}{n(n-1)} \frac{1}{\omega(n)} \sum_{i=2, n}\left[\sum_{m=1, i-1} G_{m}(p, p) G_{i-m}(q, q)+G_{m}(p, q) \cdot G_{i-m}(q, p)\right] \omega(n-i),
$$

with

$$
\omega(n)=\frac{n_{0}^{n}}{n !} \int \prod_{k=1, n} d \vec{p}_{k} S_{n}
$$

For the single-pion distribution, we are only interested in the terms of the form $\rho\left(p_{1}, p_{k}\right) \rho\left(p_{k}, p_{j}\right) \cdots \rho\left(p_{l}, p_{1}\right) C_{n-i}$ in the integrand, where $i$ is the number of $\rho$ that are not included in $C_{n-i}$. The number of this kind terms is

$$
\frac{(n-1) !}{(n-i) !}
$$

Then the single-pion distribution is

$$
P_{1}^{n}(\vec{p})=\frac{1}{n} \frac{1}{\omega(n)} \sum_{i=1, n} G_{i}(p, p) \omega(n-i) .
$$

Now the main question is to extract the expression of $\omega(n)$. From the expression of eq.(46), we have

$$
\omega(n)=\frac{1}{n} \sum_{i=1, n} i \cdot C(i) \omega(n-i)
$$


with

$$
C(i)=\frac{1}{i} \int d \vec{p} G_{i}(p, p)
$$

From the above method the two-pion and single pion inclusive distribution can be calculated for $n$ pion events.

\section{B. Two-pion correlation function for all events}

For a state $\mid \phi>$ which contains all possible multiplicities, the modified single pion distribution can be expressed as

$$
\begin{aligned}
P_{1}^{\phi}(\vec{p}) & =\frac{\left\langle\phi\left|c^{+}(\vec{p}) c(\vec{p})\right| \phi\right\rangle}{<\phi \mid \phi><M>} \\
& =\frac{\sum_{n} P_{1}^{n}(\vec{p}) \cdot n \cdot \omega(n)}{<\phi \mid \phi><M>} \\
& =\frac{\sum_{i} G_{i}(p, p) \sum_{n} \omega(n-i)}{<\phi \mid \phi><M>}=\frac{\sum_{i} G_{i}(p, p)}{<M>},
\end{aligned}
$$

where eq.(26) is applied. The two-pion inclusive distribution can be expressed as

$$
\begin{aligned}
P_{2}^{\phi}(\vec{p}, \vec{q}) & =\frac{\left\langle\phi\left|c^{+}(\vec{p}) c^{+}(\vec{q}) c(\vec{q}) c(\vec{p})\right| \phi\right\rangle}{<\phi \mid \phi><M(M-1)>} \\
& =\frac{\sum_{n} P_{2}^{n}(\vec{p}, \vec{q}) \cdot n \cdot(n-1) \omega(n)}{<\phi \mid \phi>\langle M(M-1)>} \\
& =\frac{\sum_{n}\left[\sum_{i=2, n}\left(\sum_{m=1, i-1} G_{m}(p, p) G_{i-m}(q, q)+G_{m}(p, q) \cdot G_{i-m}(q, p)\right) \omega(n-i)\right]}{<\phi \mid \phi><M(M-1)>} \\
& =\frac{\sum_{i, j} G_{i}(p, p) G_{j}(q, q)+G_{i}(p, q) \cdot G_{j}(q, p)}{<M(M-1)>} .
\end{aligned}
$$

The two-pion correlation function for all multiplicity distribution is

$$
C_{2}^{\phi}(\vec{p}, \vec{q})=\frac{P_{2}^{\phi}(\vec{p}, \vec{q})}{P_{1}^{\phi}(\vec{q}) P_{1}^{\phi}(\vec{p})} .
$$

$G_{i}(p, q), C(i)$ can be calculated either through Monte-Carlo integration or by analytical integration. 


\section{N $\pi$ CORRELATION FUNCTION FOR A PARTIALLY COHERENT SOURCE}

For a system with one coherent source and many other totally chaotic sources, the state is described by

$$
\left|\phi>_{\text {part }}=\exp \left(i \int d \vec{p} \int d^{4} x\left(j(x)+j_{c}(x)\right) \exp (i p x) c^{+}(\vec{p})\right)\right| 0>,
$$

where $c^{+}(\vec{p})$ is the pion creation operator. $j_{c}(x)$ is the current of the pion produced by coherent sources, while $j(x)$ is the current produced by totally chaotic sources, which can be expressed as eq.(3). The state $\mid \phi>_{\text {part }}$ can be expanded as

$$
\left|\phi>_{\text {part }}=\sum_{n} \frac{\left(\int\left(j_{c}(x)+j_{i n}(x)\right) e^{-i p x} c^{+}(p) d x\right)^{n}}{n !}\right| 0>=\sum \mid n>_{\text {part }},
$$

then the definition of the pion correlation function is unchanged. For n-pion state, $\mid n>_{\text {part }}$, the n-pion correlation function can be expressed as

$$
C_{n}^{\text {part }}\left(p_{1}, \cdots, p_{n}\right)=\frac{S_{n}^{\text {part }}}{\prod_{i=1, n} P_{1}^{\text {part }}\left(p_{i}\right)},
$$

with

$$
P_{1}^{\text {part }}(p)=\int g(x, p) d x+\left|j_{c}(p)\right|^{2}
$$

The $S_{n}^{p a r t}$ can be expressed as

$$
S_{n}^{\text {part }}=S_{n}\left(\rho_{I}\right)+\sum_{k=1, n} \frac{1}{k !} S_{n, k}\left(\rho_{I}, \rho_{c, k}\right)
$$

with

$$
S_{n, k}\left(\rho_{I}, \rho_{c, k}\right)=\sum_{l=1, \frac{n !}{k !(n-k) !}} S_{n}^{l}\left(\rho_{I}, \rho_{c, k}\right) .
$$

Here $S_{n}\left(\rho_{I}\right)$ is similarly defined as eq. (20) but with $\rho_{I}$ instead of $\rho$, the definition of $\rho_{I}$ is

$$
\rho_{I}\left(p_{i}, p_{j}\right)=\int d^{4} x g\left(x, \frac{p_{i}+p_{j}}{2}\right) e^{i\left(p_{i}-p_{j}\right) x} .
$$

$S_{n}^{l}\left(\rho_{I}, \rho_{c, k}\right)$ is similarly defined as $S_{n}\left(\rho_{I}\right)$ but with $k$ terms $\rho_{c}$ instead of $\rho_{I}$. Certainly, the total number of this kind of substitutions is $\frac{n !}{k !(n-k) !}$. $l$ represents one of these substitutions.

The definition of $\rho_{c}\left(p_{i}, p_{j}\right)$ is

$$
g_{c}\left(p_{i}, p_{j}\right)=j_{c}\left(p_{i}\right) j_{c}^{*}\left(p_{j}\right)
$$




\section{RESULTS AND DISCUSSIONS}

As an example, we choose the source distribution

$$
g(x, p)=\frac{1}{\left(\pi R_{0}^{2}\right)^{3 / 2}} e^{-\frac{r^{2}}{R_{0}^{2}}} \frac{1}{\left(2 \pi m_{\pi} T\right)^{3 / 2}} e^{-\frac{E}{T}} \delta(t),
$$

and its non-relativistic form

$$
g(x, p)=\frac{1}{\left(\pi R_{0}^{2}\right)^{3 / 2}} e^{-\frac{r^{2}}{R_{0}^{2}}} \frac{1}{\left(2 \pi p_{0}^{2}\right)^{3 / 2}} e^{-\frac{p^{2}}{2 p_{0}^{2}}} \delta(t),
$$

where the parameter $R_{0}$ is the radius, $\mathrm{T}$ is the temperature and $p_{0}^{2}=m_{\pi} T \cdot g\left(x, \frac{p+q}{2}\right)$ can be approximately expressed as

$$
g\left(x, \frac{p+q}{2}\right)=\frac{1}{\left(\pi R_{0}^{2}\right)^{3 / 2}} e^{-\frac{r^{2}}{R_{0}^{2}}} \frac{1}{\left(2 \pi m_{\pi} T\right)^{3 / 2}} e^{-\frac{E_{p}+E_{q}}{2 T}} \delta(t)=\frac{1}{\left(\pi R_{0}^{2}\right)^{3 / 2}} e^{-\frac{r^{2}}{R_{0}^{2}}} \frac{1}{\left(2 \pi p_{0}^{2}\right)^{3 / 2}} e^{-\frac{p^{2}+q^{2}}{4 p_{0}^{2}}} \delta(t),
$$

then we have

$$
\rho(p, q)=\int g\left(x, \frac{p+q}{2}\right) e^{i(p-q) x} d x=\frac{1}{\left(2 \pi p_{0}^{2}\right)^{3 / 2}} e^{-\frac{(p-q)^{2} R_{0}^{2}}{4}} e^{-\frac{p^{2}+q^{2}}{4 p_{0}^{2}}} .
$$

Define

$$
G_{n}(p, q)=\int \rho\left(p, p_{1}\right) \prod_{i=1, n-2} d \vec{p}_{i} \rho\left(p_{i}, p_{i+1}\right) d \vec{p}_{n-1} \rho\left(p_{n-1}, q\right)
$$

Using eq.(63), we can easily get

$$
G_{n}(p, q)=\alpha_{n} e^{-a_{n}\left(p^{2}+q^{2}\right)+g_{n} \vec{p} \cdot \vec{q}}
$$

where

$$
\begin{gathered}
a_{n+1}=a_{n}-\frac{g_{n}^{2}}{4 b_{n}}=\frac{R_{0}^{2}}{4}+\frac{1}{4 p_{0}^{2}}-\frac{R_{0}^{4}}{16 b_{n}} \\
b_{n}=a_{n}+\frac{1}{4 p_{0}^{2}}+\frac{R_{0}^{2}}{4} \\
g_{n+1}=\frac{g_{n}}{4 b_{n}}
\end{gathered}
$$


and

$$
\alpha_{n+1}=\alpha_{n}\left(\frac{1}{2 p_{0}^{2}}\right)^{3 / 2}\left(\frac{1}{b_{n}}\right)^{3 / 2}
$$

with

$$
a_{1}=\frac{R_{0}^{2}}{4}+\frac{1}{4 p_{0}^{2}}, g_{1}=R_{0}^{2} / 2 ., \alpha_{1}=\frac{1}{\left(2 \pi p_{0}^{2}\right)^{3 / 2}}
$$

Then we have

$$
C_{n}=\frac{1}{n} \int G_{n}(p, p) d \vec{p}=\alpha_{n} \frac{\pi}{n\left(2 a_{n}-g_{n}\right)^{3 / 2}} .
$$

Now we can easily calculate the multiplicity, single particle and two-particle distribution of pions according to the formula given in above sections. In fig.1, we give the pion multiplicity distribution. As the BE correlation is included, the probability with high pion multiplicity is larger than the one of usual Poissonian distribution. This feature is consistent with the nature of Bosons. We also find that as the radius and temperature decreases, the effect of BE correlation on the multiplicity distribution becomes larger.

The parameter $n_{0}$, which is the mean multiplicity without BE correlation, vs. the mean multiplicity $\left\langle M>\right.$, is given in fig. 2 . When $M$ is not very large, $n_{0}$ increases with increasing $<M>$. However, for very large $\left\langle M>\right.$, with increasing $\left\langle M>\right.$ the corresponding $n_{0}$ is almost a constant. In ref. 20 to obtain the Poissonian multiplicity distribution for a system neglecting symmetrization, a factor $\frac{n_{0}^{n}}{n !}$ has to be introduced, while in our work, starting from the classic multi-pion source the correction of multi-pion BE correlation to multiplicity distribution is obtained and it automatically reduces to a Poissonian when BE correlation is neglected.

Including the multipion BE correlation, the single particle momentum distribution for events with fixed multiplicity is given in fig.3. It is shown that, as the multiplicity of pion increases, the number of pions with low momentum becomes larger. The effect of multipion correlation on the two-pion correlation function are shown in fig.4, where we fixed the multiplicity of the events. It can be seen clearly that as the multiplicity of the event increases, 
the two-pion correlation function has a lower chaoticity, though the actual source is totally chaotic. The fitted radius also becomes smaller when multiplicity increases as shown in fig.5. This feature can be explained as follows: as the pion multiplicity increases, the emission of pions is not independent any more, multi-pion correlation is "Changed into" a factor to affect the single-pion emission probability. This gives the source some coherent property and makes the pion-source smaller. It can be seen in fig.5 that the coherent parameter has some correlations with the radius [22]. The lower coherent parameter corresponds to the smaller radius. In fig.6, two-pion correlation function with the mean multiplicity is shown. A similar property as in fig.4 can be seen. To obtain the correct behavior of the two-pion correlation function at high mean multiplicities it is very important to have correct normalization factors in eqs.(49-50), which ensure the correlation function becoming flatter and its values approaching to one, when $\langle M\rangle$ increases.

As shown in fig.2, the mean multiplicity increases very fast as $n_{0}$ approaches a certain

value. This seems to show that when $\langle M>$ becomes very large, the "hardon" picture of pion may not be suitable any more, and the system may undergo a kind of phase transition into the QGP phase. However, the increase of $\langle M\rangle$ with increasing $n_{0}$ should be very much controlled by energy conservation which is not included in our formulation. When energy conservation is included the behavior of $\left\langle M>\right.$ at large $n_{0}$ may change dramatically. Therefore, it is worthwhile to further discuss the effects of multi-pion correlation on various observables after including energy conservation [23].

\section{Acknowledgement}

We wish to express our gratitude to Dr. Pang Yang for helpful discussion. 


\section{REFERENCES}

[1] R. Hanbury-Brown and R. Q. Twiss, Nature (London) 178 (1956)1046

[2] G. Goldhaber et al., Phys. Rev. 120 (1960) 300

[3] D. H. Boal, C. K. Gelbke and B. K. Jenings, Rev. Mod. Phys. V62 (1990) 553

[4] W. A. Zajc, in Hadronic Multiparticle Production, ed. P. Carruthers (World Scientific, Singapore, 1987)p. 125

[5] B. Lorstad, Int. J. Mod. Phys. A4 (1988) 2861

[6] M. Gyulassy, S. K. Kauffmann and Lance Wilson, Phys. Rev. C 20 (1979) 2267

[7] G. Goldhaber, in local Equilibrium in Strong Interaction Physics, Proccedings of the First Workshop, Bad Houston, West Germany, 1984, edited by D. K. Scott and R. M. Weiner (World Scientific, Singapore,1985)

[8] W. Willis and C. Chasman, Nucl. Phys. A418 (1984) 425c.

[9] W. A. Zajc, Phys. Rev. D35 (1987) 3396.

[10] Y. M. Liu et al., Phys. Rev. C34 (1986) 1667.

[11] M. Biyajima, Prog. Theor. Phys. 66 (1981) 1378.

[12] R. M. Weiner, Phys. Lett. B232(1989)278; ibid. B242(1990)547.

[13] W. N. Zhang et. al, Phys. Rev. C47 (1993) 795.

[14] John G. Cramer, Phys. Rev. C43 (1991) 2798.

[15] NA35 Collab.. T. J. Humanic, Z. Phys. C38 (1988) 79.

[16] WA80 Collab.. Nucl. Phys. A525 (1991) 333c.

[17] T. Abbott et al., Nucl. Phys. A544(1992)237.

[18] NA44 Collab.. preprint CERN-PPE/94-75 to be published. 
[19] William. J. Knox, Phys. Rev. D10, 65 (1974).

[20] S. Pratt, preprint MSUCL-867(1992); Phys. Lett. 310B (1993) 159.

[21] W. Q. Chao, C. S. Gao, and Q. H. Zhang; Phys. Rev. C49 (1994) 3224.

[22] Q. H. Zhang and W. Q. Chao, Commun. theor. Phys. V22 (1994)187.

[23] W. Q. Chao, C. S. Gao, and Q. H. Zhang; in preparation 


\section{Figure Captions}

1. Pion multiplicity distribution with (dot-dashed cure) and without (solid cure) BoseEinstein correlations, the input value of $R, p_{0}$ and $n_{0}$ is $3 \mathrm{fm}, 0.18 \mathrm{GeV}$ and 13 respectively.

2. $n_{0}$ vs. the mean multiplicity $M$. The dot-dashed line and solid line correspond to the case with and without BE correlation. The input value of $R$ and $p_{0}$ is $5 \mathrm{fm}$ and $0.25 \mathrm{GeV}$ respectively.

3. The single particle momentum distribution for different multiplicities. the dashed line, dot-dashed line and dotted line corresponds to multiplicity $M=100,200$ and 400 respectively. The solid line corresponds to the input momentum distribution. The input value of $R$ and $p_{0}$ is $5 \mathrm{fm}$ and $0.25 \mathrm{GeV}$ respectively.

4. Two-pion correlation function for fixed multiplicity events. The solid line, dashed line and dot-dashed line correspond to multiplicity $M=100,200$ and 400 . The input value of $R$ and $p_{0}$ is $4.95 \mathrm{fm}$ and $0.25 \mathrm{GeV}$ respectively.

5. The effective coherent parameter $\lambda$ (dashed line) and radius $R$ (solid line) vs. multiplicity $M$. The input value of $R$ and $p_{0}$ is $4.95 \mathrm{fm}$ and $0.25 \mathrm{GeV}$ respectively.

6. Two-pion correlation function for all events. The solid line, dashed line, dot-dashed line and dotted line correspond to mean multiplicity $\langle M>=5,26,107$ and 180 . The input value of $R$ and $p_{0}$ is $4.95 \mathrm{fm}$ and $0.25 \mathrm{GeV}$ respectively. 